\title{
Sarcoidosis Presenting with Bilateral Optic Disc Edema as the Initial Presentation
}

\author{
Merve Beyza Yildiz ${ }^{1}$, Sevcan Balci ${ }^{1}$, Sehnaz Ozcaliskan² and Ece Turan Vural ${ }^{1}$ \\ ${ }^{1}$ Department of Ophthalmology, Haydarpasa Numune Training and Research Hospital, University of Health Sciences, Istanbul, Turkey \\ ${ }^{2}$ Department of Ophthalmology, Beyoglu Eye Training and Research Hospital, University of Health Sciences, Istanbul, Turkey
}

\begin{abstract}
Sarcoidosis is a chronic, progressive, granulomatous inflammatory disease of unknown etiology that affects multiple organ systems. Granulomatous anterior uveitis is the most common eye finding, and optic nerve involvement at presentation is rarely seen. Here, we report a 43-year man referred to our clinic with eye pain, decreased vision, floaters and bilateral papillitis, vitritis and cystoid macular edema. The level of serum angiotensin-converting enzyme, thoracic computed tomography and transbronchial needle aspiration biopsy of lymph nodes were confirmatory for sarcoidosis. In this case, sarcoidosis initially presented with bilateral papillitis and vitritis without granulomatous uveitis, and successful results were obtained with methylprednisolone and immunosuppressive treatment without any relapse or systemic involvement of sarcoidosis. Sarcoidosis should be considered in patients presenting with optic nerve inflammation, even when granulomatous inflammation or vasculitis are not present, which are specific for ocular sarcoidosis.
\end{abstract}

Key Words: Edema, Optic disc, Papillitis, Sarcoidosis.

How to cite this article: Yildiz MB, Balci S, Ozcaliskan S, Vural ET. Sarcoidosis Presenting with Bilateral Optic Disc Edema as the Initial Presentation. J Coll Physicians Surg Pak 2021; 31(07):861-863.

\section{INTRODUCTION}

When all causes of uveitis are considered, sarcoidosis is seen in $3-7 \%$ of all, and ocular involvement is present in $30-60 \%$ of all patients with sarcoidosis. Granulomatous anterior uveitis is the most common presentation with a rate of $25 \%$ of all cases. ${ }^{1}$

Sarcoidosis may affect the optic nerve in various ways. Optic disc edema due to posterior uveitis, elevated intraocular pressure, optic neuropathy, optic atrophy secondary to compression or infiltration by primary central nervous system lesion, and primary granuloma of optic nerve head are the optic nerve pathologies that can be seen insarcoidosis. ${ }^{2}$

Leading causes of optic disc swelling are elevated intracranial pressure (papilledema), inflammatory diseases, optic neuritis, optic neuropathies including ischemic, infiltrative, compressive, toxic, hereditary, paraneoplastic optic neuropathy, congenital optic disc anomalies and other vascular diseases; and the differential diagnosis of optic disc swelling is crucial because of its systemic associations with life-threatening conditions. ${ }^{3}$ The term papillitis is used when the optic nerve is affected with severe inflammation.

Correspondence to: Dr. Merve Beyza Yildiz, Department of Ophthalmology, Haydarpasa Numune Training and Research Hospital, University of Health Sciences,

Istanbul, Turkey

E-mail: mervebeyza_afl@hotmail.com

Received: September 17, 2019; Revised: December 26, 2019;

Accepted: December 30, 2019

DOI: https://doi.org/10.29271/jcpsp.2021.07.861
We report a case of sarcoidosis, presenting initially with bilateral papillitis and vitritis; and the diagnosis was confirmed after systemic investigations.

\section{CASE REPORT}

A 43-year man was referred to our clinic with eye pain, redness, decreased vision, and floaters bilaterally, which were presentfor a month. He had no systemic symptoms, diseases, medication use or relevant family history. His best-corrected visual acuity (BCVA) was 20/100 bilaterally. Intraocular pressure with Goldmann applanation tonometry was 11 and $12 \mathrm{mmHg}$ in his right and left eyes, respectively. Biomicroscopic examination revealed posterior synechia with + 3 cells in the vitreous; and on fundoscopic evaluation, hyperemic and swollen optic discs were present in both eyes (Figure 1a). Fundus fluorescein angiography (FFA) demonstrated focal leakage in the macula with fluorescein leakage and staining of the optic disc and optical coherence tomography (OCT) imaging of the macula showed hyporeflective intraretinal cystoid spaces in the right and left eyes, which were indicative of inflammation (Figure 1b, 1c). After comprehensive laboratory tests, including serological panel, infectious diseases were excluded. He was consulted to neurology, pulmonology and internal medicine departments. Brain, orbital magnetic resonance imaging (MRI) and lumbar puncture (LP) findings were normal. Laboratory tests revealed elevated levels of serum angiotensin-converting enzyme (111 U/L), and chest computed tomography (CT) showed enlargement of paratracheal and subcarinal lymph nodes. Histopathology of transbronchial needle aspiration biopsy performed by a pulmonologist confirmed the diagnosis of sarcoidosis. 


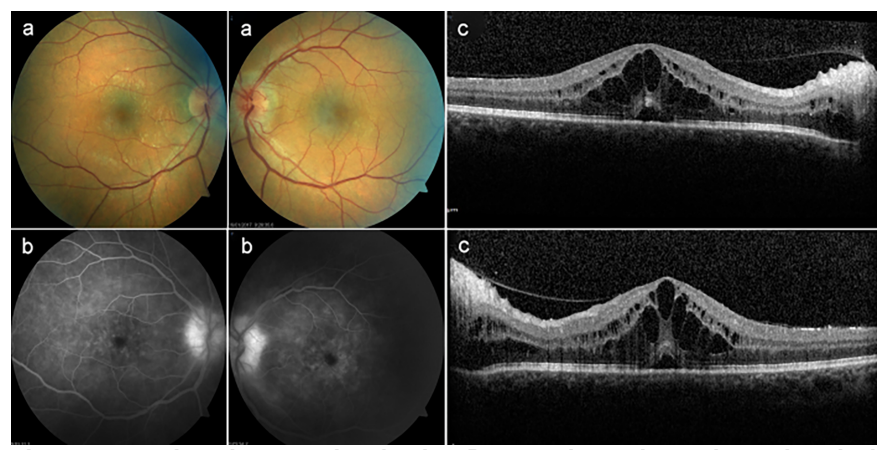

Figure 1: Fundus photography, fundus fluorescein angiography and optical coherence tomography at presentation: (a) The fundus examination showed hyperemia and swelling of the optic discbilaterally. (b) Fundus fluorescein angiography showed bilateral focal leakage in the macula, fluorescein leakage and staining of both optic discs. (c) Optical coherence tomography was performed, which revealed a cystoid macularedema in both eyes.
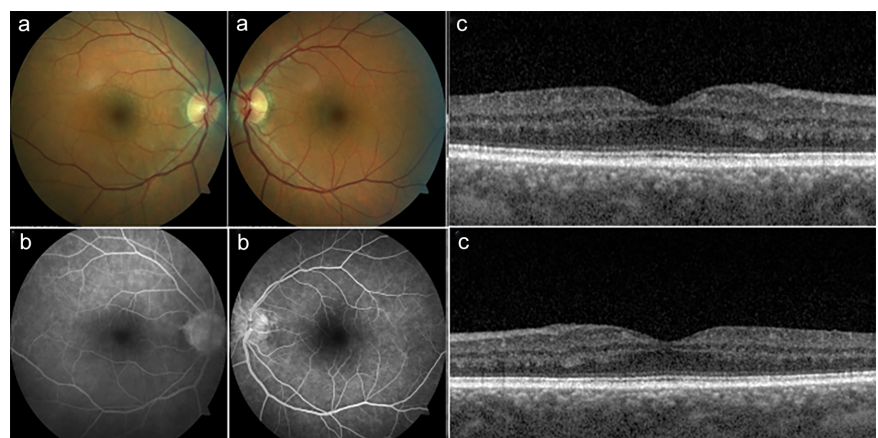

Figure 2: Fundus photography, fundus fluorescein angiography and optical coherence tomography at six months after treatment: (a) The fundus examination showed that the optic disc edema has resolved with pallor in the temporal region of the left eye. (b) In the fundus fluorescein angiography, there was no leakage and staining in the macula and optic disc bilaterally. (c) Cystoid macularedema improved in botheyes.

The patient was treated with intravenous pulse methylprednisolone, $1000 \mathrm{mg}$, for three consecutive days, followed by oral methylprednisolone, $1 \mathrm{mg} / \mathrm{kg} /$ day, daily with weekly tapering. Topical prednisolone acetate $1 \%$ and cyclopentolate hydrochloride $1 \%$ were also administered. At one month, due to the progression of the macular edema, intravitreal dexamethasone implant was applied to both eyes with oral methotrexate, $15 \mathrm{mg}$, and folic acid, $5 \mathrm{mg}$, weekly. At the $6^{\text {th }}$ month follow-up examination, his BCVA improved to 20/20, and no active inflammation was observed in the anterior chamber and vitreous bilaterally. Optic disc edema was resolved with minimal pallor in the temporal region (Figure $2 a)$, and automated perimetry showed generalised reduction in sensitivity with scotomas in the left eye. In the FFA performed on follow-up examination, no leakage or staining was observed in the macula and optic disc bilaterally. In the OCT imaging, cystoid macular edema was resolved (Figure $2 b, 2 c)$. During 12 months follow-up, ophthalmological examinations were stable. Thoracic computed tomography findings were resolved, and no recurrence and other systemic involvement of sarcoidosis was observed.
Ocular sarcoidosis usually presents as granulomatous anterior uveitis. Optic nerve involvement has been reported in $25-60 \%$ of all sarcoidosis cases. ${ }^{4}$ The initial presentation is variable in sarcoidosis. There are a few case reports that emphasise sarcoidosis cases initially presenting with papillitis. ${ }^{5,6}$ In sarcoidosis, optic disc edema may occur as an isolated granulomatous inflammation of the optic nerve, or the disease may involve visual pathways, particularly optic chiasm and meninges.

The interesting part of our case is that vitritis and papillitis were the only clinical presentations without granulomatous uveitis and central nervous system involvement and that sarcoidosis was diagnosed after systemic investigation and successful results were obtained with high dose cortisone and immunosuppressive treatment.

Due to its association with life-threatening systemic conditions, accurate differentiation of optic disc swelling is essential. In our case, the patient had bilateral posterior synechia and +3 cells in the vitreus, and these findings led us to investigate the inflammatory diseases.

Similarly, neurosarcoidosis should be ruled out in patients presenting with optic disc swelling. We did not observe any evidence relevant to neurosarcoidosis with MRI and LP investigations.

In conclusion, early diagnosis and initiation of treatment can prevent visual loss before inflammation permanently affects the optic nerve and disease progresses to multiple system involvement.

\section{PATIENT'S CONSENT:}

Written informed consent was obtained from the patient for publication of this case report and accompanying images.

\section{CONFLICT OF INTEREST:}

The authors declared no conflict of interest.

\section{AUTHORS' CONTRIBUTION:}

MBY, SB, SO, ETV: Contributed to the design and data collection, literature review and the writing of the manuscript.

\section{REFERENCES}

1. Akova AY, Kadayıfçılar S, Aydın P. Sarkoidoz. Ret-Vit 2000; 8:102-113.

2. Ohara K, Okubo A, Sasaki H, Kamata K. Intraocular manifestations of systemic sarcoidosis. Jpn J Ophthalmol 1992; 36:452-7.

3. Miller NR, Newman NJ, eds. Walsh \& Hoyt's clinical neuroophthalmology: The essentials 2 nd ed. Lippincott Williams \& Wilkins 2008; 122-145.

4. James DG, Zatouroff MA, Trowell J, Rose FC. Papilloedema in sarcoidosis. Br J Ophthalmol 1967; 51(8): 526-9. doi: 10.1136/bjo.51.8.526.

5. Austin AL, Day LT, Bishop FM. Acute vision loss: A fuzzy presentation of sarcoidosis. J Emerg Med 2013; 44(4): 
e325-8. doi: 10.1016/j.jemermed.2012.11.007.

6. Caiyun $Y$, Lina M, Stephen D. Anesi. Bilateral papillitis and vitritis as the initial ophthalmologic finding in a patient with complex medical history, leading to diagnosis of multisystem sarcoidosi. Am J Ophthalmol Case Rep 2018; 13:122-6. doi: 10.1016/j.ajoc.2018. 12.020. eCollection

$\bullet \bullet \bullet \bullet \bullet \bullet \bullet \bullet \bullet$ 